\title{
Níveis de resíduo de cevada na dieta de frangos de corte Label Rouge
}

\section{Barley residue levels in the diet of Label Rouge broilers}

Universidade Federal do Mato Grosso (UFMT), Cuiabá, MT, Brasil

* Correspondência: alnm.luz@gmail.com

Submetido: 18 ago 2021 | Aprovado: 8 dez 2021

DOI: http://dx.doi.org/10.7213/acad.2022.20002

Rev. Acad. Ciênc. Anim. 2022;20:e20002

\author{
Anna Luz Netto Malhado (i) \\ Júlia Rodrigues Alves (D \\ Heder José D'avilla Lima (D) \\ Mauricio Silva Rosa (D)
}

\section{Resumo}

Objetivou-se com este trabalho avaliar o desempenho zootécnico de frangos Label Rouge alimentados com diferentes níveis de resíduo de cevada em substituição ao milho. Foram utilizadas 140 aves, sendo as rações experimentais formuladas à base de milho e farelo de soja e os tratamentos constituídos por quatro níveis de resíduo de cevada $(0,4,8$ e 12\%), sendo cinco repetições com sete animais cada. Os parâmetros avaliados foram: consumo de ração, conversão alimentar, peso final, ganho de peso e rendimento de carcaça, cortes, vísceras e gordura. Observou-se maior consumo de ração conforme aumentou-se o nível de inclusão de resíduo de cevada na dieta dos animais. Não foram observadas diferenças significativas para conversão alimentar, peso final, ganho de peso, rendimento de carcaça, cortes comerciais, vísceras e gordura. A variável de rendimento de dorso apresentou efeito quadrático, sendo que - nível estimado de inclusão que causou o menor rendimento foi o de $2,5 \%$. O uso de resíduo de cevada na ração não prejudicou o desempenho zootécnico e o rendimento de carcaça, corte e vísceras de aves de corte Label Rouge criadas em sistema de confinamento.

Palavras-chave: Alimento alternativo. Fibra. Frango caipira.

\begin{abstract}
The objective of this work was to evaluate the zootechnical performance of Label Rouge broilers fed different barley residue levels in substitution to corn. A total of 140 birds were used. The experimental rations were based on corn and soybean meal, and the treatments consisted of four barley residue levels (0, 4, 8 and 12\%), five replicates with seven animals each. The parameters evaluated were: food intake, food conversion, final weight, weight gain, carcass yield, cuts, viscera and fat. Higher feed intake was observed as the level of inclusion of barley residue in the animals' diet was increased. No significant differences were observed for feed conversion, final weight, weight gain, carcass yield, commercial cuts, viscera and fat. The back yield variable presented a quadratic effect, and the estimated level of inclusion that caused the lowest income was $2.5 \%$. The use of barley residue in the feed did not affect the performance and carcass yield, cut and offal of Label Rouge cutting birds kept in a feedlot system.
\end{abstract}

Keywords: Alternative food. Fiber. Chicken. 


\section{Introdução}

A avicultura brasileira tem se destacado mundialmente pela produtividade e qualidade da carne produzida. O Brasil é o terceiro maior produtor e maior exportador de carne de frango, totalizando 13,845 milhões de toneladas produzidas em 2020 e consumo per capita em média de 45,27 quilos por habitante ao ano (ABPA, 2021). Tendo em vista a demanda dos consumidores por produtos mais saborosos, firmes e com sabor pronunciado (Madeira et al., 2010), a criação de aves de desempenho lento ganha espaço no mercado interno, principalmente de pequenos e médios produtores. Estimativas do Ministério do Desenvolvimento Agrário (MDA) indicam que a avicultura caipira de subsistência gera acesso à alimentação de $80 \%$ dos produtores familiares (ABNT, 2015).

Dentre as raças de frangos de desempenho lento criadas no Brasil, destaca-se a Label Rouge, também conhecida como pescoço pelado vermelho. Trata-se de um animal rústico e de fácil manejo, plumagem avermelhada, bico e pés amarelados e ausência de penas no pescoço, tanto nos machos quanto nas fêmeas, com pele fina e sem acúmulo de gordura, sabor e textura inconfundíveis, sendo a raça de ave tipo caipira mais criada no Brasil e na França (Avifran, 2016).

Na indústria avícola, a alimentação é o componente de maior importância, representando cerca de 75\% do custo de produção, sendo o milho e o farelo de soja os ingredientes mais comuns na dieta desses animais. Tendo em vista a variação do preço desses ingredientes, a competitividade com outras culturas, a produção de biodiesel e óleos e o consumo humano, pesquisadores, técnicos e produtores estão sempre à procura de alimentos alternativos que possam reduzir os custos de produção e manter a eficiência produtiva e a qualidade nutricional.

A cevada (Hordeumvulgare sp.vulgare) é um cereal importante na produção de malte e na alimentação humana e animal (Carreira, 2011). A maior parte da cevada produzida no Brasil é utilizada nas indústrias de bebidas (cerveja e destilados), gerando diversos coprodutos como o resíduo de cevada. Esse resíduo representa cerca de $85 \%$ dos coprodutos gerados nas indústrias cervejeiras, portanto, sua utilização na alimentação animal como ingrediente alternativo na substituição total ou parcial do milho e outros cereais é de suma importância, principalmente em zonas ou épocas de escassez (Costa et al., 2006; Carreira, 2011), tornando-se uma possível alternativa para a redução do custo da ração.

Apesar de suas qualidades nutricionais, o resíduo de cevada tem sua utilização restrita para animais monogástricos devido ao seu elevado teor de fibra bruta, que diminui a digestibilidade dos alimentos, e pela presença de polissacarídeos não-amiláceos (PNAs) que, principalmente em aves, atuam como fatores antinutricionais, aumentando a viscosidade da digesta no intestino, o que prejudica a digestão e absorção de nutrientes, resultando em uma baixa performance dos animais e no surgimento de problemas intestinais. No entanto a utilização de alimentos com maiores níveis de fibra bruta, como é o caso da cevada (resíduo de cervejaria ou bagaço de cevada), no sistema de criação de aves de desempenho lento, como o Label Rouge, torna-se interessante à medida em que essas aves possuem melhor capacidade de digerir fibra.

Levando-se em conta a necessidade de pesquisas com fontes alternativas de alimentos para a dieta de aves de corte e a escassez de informação quanto à utilização de cevada na dieta de frangos Label Rouge, objetivou-se com esse estudo avaliar os efeitos de diferentes níveis de inclusão de resíduo de cevada em substituição ao milho sobre o desempenho e características de carcaça de frangos de corte Label Rouge dos 49 aos 90 dias de idade.

\section{Material e métodos}

Esta pesquisa foi aprovada pelo Comitê de Ética no Uso de Animais, da Universidade Federal do Mato Grosso (UFMT), protocolo $n^{\circ} 23108.007748 / 14-2$.

O experimento foi conduzido no setor de Avicultura da Fazenda Experimental da UFMT, campus de Cuiabá, localizada no distrito de Santo Antônio do Leverger. Foram utilizadas 140 aves machos e fêmeas da raça Label Rouge, dos 49 aos 90 dias de idade, distribuídas em delineamento inteiramente casualizado, com quatro tratamentos, cinco repetições e sete animais por repetição, totalizando 20 unidades experimentais. Os tratamentos foram: 0, 4, 8 e 12\% de resíduo de cevada em substituição ao milho. 
As aves foram alojadas em galpões de alvenaria, subdividos em boxes, cobertos por telhas de barro e equipados com ventiladores e aspersores. Cada unidade experimental foi equipada com um bebedouro pendular automático e um comedouro tubular semiautomático com capacidade para $15 \mathrm{~kg}$. Os animais receberam rações isoenergéticas e isoproteicas, e água ad libtum. As dietas experimentais foram formuladas para atender às exigências nutricionais preconizadas para frangos de desempenho lento na fase de crescimento e terminação de acordo com Rostagno et al. (2011) e com as tabelas de composição nutricional da Fundación Española para el Desarrollo de la Nutrición Animal (Calsamiglia et al., 2016) (Tabela 1).

Aos 90 dias de idade, duas aves por repetição foram selecionadas aleatoriamente, insensibilizadas e abatidas pelo procedimento de sangria. Posteriormente, os cortes nobres foram separados e pesados a fim de se obter os rendimentos de cortes. Realizou-se jejum pré-abate de 8 horas para evitar contaminação da carcaça com resíduos gastrointestinais.

Tabela 1 - Composição percentual e calculada das rações experimentais, na base da matéria natural

\begin{tabular}{lcccc}
\hline \multirow{2}{*}{ Ingredientes (\%) } & \multicolumn{3}{c}{ Níveis de bagaço de cevada (\%) } \\
\cline { 2 - 5 } & $\mathbf{0}$ & $\mathbf{4}$ & $\mathbf{8}$ & $\mathbf{1 2}$ \\
\hline Milho moído & 57,90 & 52,50 & 47,00 & 41,50 \\
Farelo de soja (45\%) & 37,03 & 37,03 & 37,03 & 37,03 \\
Óleo de soja & 1,51 & 1,51 & 1,51 & 1,51 \\
Calcário calcítico & 0,76 & 0,76 & 0,76 & 0,76 \\
Fosfato bicálcico & 1,25 & 1,25 & 1,25 & 1,25 \\
Sal comum & 0,45 & 0,45 & 0,45 & 0,45 \\
Núcleo & 1,00 & 1,00 & 1,00 & 1,00 \\
Cevada & 0,00 & 4,00 & 8,00 & 12,00 \\
Amido & 0,10 & 1,50 & 3,00 & 4,50 \\
Composição calculada & & & 2950 \\
Energia metabolizável (kcal/kg) & 2950 & 2950 & 2950 & 20,00 \\
Proteína bruta (\%) & 20,00 & 20,00 & 20,00 & 1,05 \\
Lisina digestível (\%) & 1,05 & 1,05 & 1,05 & 0,65 \\
Metionina + Cistina digestível (\%) & 0,65 & 0,65 & 0,65 & 0,24 \\
Triptofano digestível (\%) & 0,24 & 0,24 & 0,24 & 0,73 \\
Treonina digestível (\%) & 0,73 & 0,73 & 0,73 & 0,70 \\
Cálcio (\%) & 0,70 & 0,70 & 0,70 & 0,34 \\
Fósforo disponível (\%) & 0,34 & 0,34 & 0,34 & 0,19 \\
Sódio (\%) & 0,19 & 0,19 & 3,26 \\
Fibra bruta (\%) & 2,85 & 3,09 & \\
\hline
\end{tabular}

Nota: ${ }^{1}$ Composição/kg de produto: Cálcio: 150g, Fósforo: 50g, Metionina: 33g, Cobre: 160g, Cobalto: 2g, Ferro: 630mg, lodo: 20mg ${ }^{2}$ Composição/kg de produto: Vit. A:100.000 U.I., Vit D3:50.000 U.I., Vit. E: 330 U.I., Vit B1 :40 mg, Vit. B12: 220mcg, Selênio: 6,5mg, Vit B2: 100 mg, Vit B6:44 mg, Biotina: 200 mg, Vit. K3: 22 mg, Ácido fólico: 11 mg, Niacina: 770 mg, Pantotenato de cálcio: 220 mg, Colina: 5.000 mg, Sódio: 26 g, Manganês: 1.600 mg, Zinco: 1.300 mg, Fitase: 10.000 FTU, Avilamicina: 150 mg, Salinomicina1.320 mg. 
Os dados de desempenho (consumo diário de ração, ganho de peso, peso final e conversão alimentar) foram obtidos semanalmente através da pesagem dos animais e quantificação das sobras das rações. $O$ rendimento de carcaça foi determinado pela relação do peso da carcaça eviscerada e peso das aves na ocasião do abate, sendo calculado da seguinte forma: [\%RC $=($ peso carcaça $\times 100) /$ peso vivo]. Os cortes foram separados em peito, coxa, sobrecoxa, asas, dorso (cabeça + pescoço), pés e vísceras comestíveis (moela, fígado e coração). A gordura abdominal (tecido adiposo ao redor da bursa de Fabricius, proventrículo, moela e cloaca) foi separada e pesada para análise de rendimento. Para calcular o rendimento destes em relação à carcaça, utilizou-se a fórmula: [\%R do corte ou vísceras ou gordura $=$ (peso cortes ou vísceras ou gordura $\times 100$ ) /peso carcaça].

Os dados foram analisados estatisticamente, utilizando o programa ASSISTAT 7.7 - Universidade Federal de Campina Grande, Brasil.

\section{Resultados e discussão}

Não foram observadas diferenças significativas $(p>0,05)$ para ganho em peso, peso final e conversão alimentar, como demonstrado na Tabela 2. Para o consumo de ração, observou-se diferença significativa $(p<0,05)$ com efeito linear crescente, onde $o$ aumento na inclusão de cevada na dieta dos animais proporcionou maior consumo de ração. $O$ aumento no consumo de ração pelos animais pode ser explicado pela presença de PNAs na cevada, o que pode ter ocasionado o aumento da viscosidade da digesta, tornando os nutrientes menos disponíveis no trato digestório, sendo o aumento no consumo de ração a forma de se aumentar a disponibilidade desses nutrientes e suprir a exigência nutricional das aves.

Parpinelli (2016), trabalhando com a inclusão de resíduo seco de cervejaria $(0,2,4,6,8$ e 10\%) na dieta de frangos de corte Cobb 500 na fase de 1-21 dias e 1-42 dias de idade, não encontrou diferença $(p>0,05)$ entre os tratamentos para as variáveis de desempenho zootécnico avaliadas, consumo de ração, ganho em peso e conversão alimentar, divergindo dos resultados encontrados no presente trabalho. Os resultados encontrados podem ser explicados pela ação das leveduras presentes no resíduo seco de cervejaria (RSC), que minimizaram os efeitos antinutricionais dos altos teores de fibra, além de serem uma fonte de biomassa de alta qualidade.

Resultados distintos aos encontrados neste trabaTho para consumo de ração foram verificados por Oliveira et al. (2005). Os autores avaliaram o nível de inclusão de duas granulometrias de resíduo de cervejaria desidratado para frangos de corte Cobb Avian 48 na fase de 1 a 14 dias de idade, não encontrando diferenças significativas $(p>0,05)$ para as variáveis avaliadas de consumo de ração, ganho de peso e conversão alimentar, porém observaram uma diminuição numérica no consumo de ração à medida que se aumentava a inclusão de resíduo de cervejaria e óleo na dieta.

Tabela 2 - Desempenho de frangos de corte Label Rouge aos 90 dias de idade

\begin{tabular}{lccccc}
\hline \multirow{2}{*}{ Parâmetros } & \multicolumn{5}{c}{ Níveis de cevada (\%) } \\
\cline { 2 - 6 } & $\mathbf{0}$ & $\mathbf{4}$ & $\mathbf{8}$ & $\mathbf{1 2}$ & $\mathrm{CV}(\%)$ \\
\hline $\mathrm{CR}^{\star}(\mathrm{g} / \mathrm{dia})$ & 137,3 & 140,0 & 142,1 & 144,5 & 3,2 \\
$\mathrm{GP}^{\text {ns }}(\mathrm{g})$ & 1480,0 & 1591,6 & 1235,2 & 1472,7 & 15,3 \\
$\mathrm{PF}^{\text {ns }}(\mathrm{g})$ & 2357,8 & 2456,0 & 2096,0 & 2337,5 & 14,6 \\
$\mathrm{CA}^{\mathrm{ns}}(\mathrm{g} / \mathrm{g})$ & 3,8 & 3,6 & 4,7 & 4,0 & 15,8 \\
\hline
\end{tabular}

Nota: $\mathrm{CR}=$ consumo de ração; $\mathrm{GP}=$ ganho de peso; $\mathrm{PF}=$ peso final; $\mathrm{CA}=$ conversão alimentar; $\mathrm{ns}=$ não significativo ao nível de $5 \%$ de probabilidade $(p>0,05)$. *Significativo ao nível de $5 \%$ de probabilidade $(p<0,05)$. Equação de regressão linear: $y=137.43972000+$ $0.59020500 . x ; r^{2}=99 \%$. 
Rosin (2012) avaliou a inclusão de cevada na dieta de frangos de corte da linhagem Cobb 500, com idade de 8 a 21 dias de idade, com níveis de 5 , 10 e $15 \%$ de inclusão de cevada em substituição ao milho e observou efeito linear decrescente sobre o peso final, ganho de peso e consumo de ração. Tais resultados distintos aos encontrados neste trabalho podem ser explicados pelo fato de os animais Cobb Avian 48 e Cobb 500 estarem em fase inicial de crescimento, com menor capacidade de digestão nesta fase, pois o trato gastrintestinal não está totalmente desenvolvido, enquanto no presente estudo os animais Label Rouge se encontravam em fase final de crescimento e possuíam maior capacidade de digestão dos alimentos e de fibras por conta do hábito de pastejo, consumo de forragens e alimentos naturais e fibrosos quando criados em sistema extensivo ou semi-intensivo (criação caipira).

Ao avaliar os animais na fase de 21 a 42 dias de idade, Parpinelli (2016) encontrou efeito para consumo de ração, ganho de peso e conversão alimentar quando feita a inclusão do resíduo seco de cervejaria na forma de níveis crescentes $(0,2,4,6,8$ e $10 \%)$, sendo observado efeito linear para os valores de ganho de peso e conversão alimentar.

Devido a seu sistema gastrintestinal mais desenvolvido para a digestão de fibras, os frangos de desempenho lento têm maior capacidade que os frangos de corte comerciais de converter alimentos de menor qualidade em carne. Essa vantagem se deve à maior capacidade de trituração da moela e da flora microbiana no ceco (Barbosa et al., 2007). O aumento no consumo de ração pode ser explicado pelo sistema adotado (intensivo) e pelo fato de a fibra ter sido fornecida na dieta em forma de ração farelada.

O rendimento de carcaça, cortes comerciais, vísceras comestíveis e gordura não tiveram diferença estatística entre si $(p>0,05)$ m os mesmos para todos os tratamentos. O rendimento de dorso, contudo, apresentou efeito quadrático $(p<0,05)$ (Tabela 3 ), sendo que o nível estimado que causou o menor rendimento de dorso foi o de $2,5 \%$ de inclusão de cevada.

Tabela 3 - Rendimento de carcaça, cortes, vísceras e gordura de frangos de corte Label Rouge abatidos aos 90 dias de idade

\begin{tabular}{|c|c|c|c|c|c|}
\hline \multirow{2}{*}{ Parâmetros (\%) } & \multicolumn{5}{|c|}{ Níveis de Cevada (\%) } \\
\hline & 0 & 4 & 8 & 12 & $\mathrm{CV}$ \\
\hline Rendimento de carcaçans & 80,2 & 74,8 & 78,5 & 78,3 & 5,3 \\
\hline Rendimento de peito ${ }^{\text {ns }}$ & 18,8 & 18,4 & 18,0 & 18,1 & 10,7 \\
\hline Rendimento coxa + sobrecoxa ${ }^{\text {ns }}$ & 22,1 & 21,2 & 21,9 & 21,8 & 7,6 \\
\hline Rendimento de asans & 9,1 & 8,7 & 9,4 & 9,0 & 8,4 \\
\hline Rendimento do dorso* & 24,7 & 22,4 & 24,4 & 24,9 & 7,3 \\
\hline Rendimento dos pés ${ }^{\text {ns }}$ & 4,3 & 3,7 & 4,5 & 4,1 & 14,8 \\
\hline Rendimento do fígadons & 1,5 & 1,5 & 1,6 & 1,7 & 20,3 \\
\hline Rendimento do coraçãons & 0,5 & 0,4 & 0,5 & 0,5 & 24,8 \\
\hline Rendimento da moela ${ }^{\text {ns }}$ & 1,9 & 1,8 & 2,2 & 2,0 & 18,1 \\
\hline Rendimento de gordurans & 1,2 & 1,7 & 1,2 & 1,2 & 82,7 \\
\hline
\end{tabular}

Nota: $n s=$ não significativo ao nível de $5 \%$ de probabilidade $(p>0,05)$ * Significativo ao nível de 5\% de probabilidade ( $p<0,05)$. Equação de regressão quadrática: $y=24.66000000-1.58041667 . x+0.31125000 . x^{\wedge} 2-0.01481771 . x^{\wedge} 3 ; r^{2}=99,99 \%$.

Parpinelli (2016), ao avaliar os valores encontrados para rendimentos de carcaça e cortes nobres aos 21 dias, não encontrou alterações em resposta à inclusão de resíduo seco de cervejaria $(p>0,05)$, sendo o mesmo demonstrado no presente trabalho. $\mathrm{O}$ autor verificou efeito dos níveis de inclusão de RSC sobre o rendimento de órgãos, como coração e intestino, e porcentagem de gordura abdominal, diferindo dos resultados encontrados na Tabela 3, onde não há diferenças significativas entre as variáveis 
avaliadas. Segundo Parpinelli (2016), o rendimento de coração acompanhou o rendimento de carcaça de forma linear à medida que se aumentava o nível de inclusão do resíduo seco de cervejaria, sugerindo que com o aumento do desenvolvimento das aves, estas necessitariam de maior aporte sanguíneo e nutrientes, gerando assim um aumento desse órgão como compensação.

Ao avaliar o rendimento de carcaça e cortes nobres das aves aos 42 dias, Parpinelli (2016) demonstrou que não houve interferência $(p>0,05)$ do RSC sobre o desenvolvimento corporal das aves, rendimento de cortes e de carne do peito, pesos relativos de órgãos (fígado, coração, moela e intestino) e de gordura abdominal para os diferentes níveis de inclusão de RSC, corroborando com os resultados encontrados no presente trabalho, onde a inclusão de resíduo de cevada na dieta de aves Label Rouge não interferiu na produção e no desenvolvimento zootécnico quando incluída até o nível de $12 \%$ na dieta em substituição ao milho.

\section{Conclusão}

O resíduo de cevada pode ser utilizado com inclusão $12 \%$ na dieta de frangos de corte Label Rouge, dos 49 aos 90 dias de idade, sem causar reduções no desempenho zootécnico.

\section{Referências}

ABPA - Associação Brasileira de Proteína Animal. Produção de carne de frango totaliza 13,146 milhões de toneladas em 2015. 2016 [acesso 5 set 2016]. Disponível em: https://tinyurl. com/5byyjcvv

ABNT - Associação Brasileira de Normas Técnicas. ABNT NBR 16389 - Produção, abate, proces samento e identificação do frango caipira, colonial ou capoeira. ABNT; 2015.

Avifran. Guia de raças; 2016 [acesso 5 set 2020]. Disponível em: http://www.avifran.com.br/portifolio-detalhes.php?acao=6

Barbosa FJV, Nascimento MPSB, Diniz FM, Nascimento HTS, Araújo Neto RB. Sistema alternativo de criação de galinhas caipiras. Teresina: Embrapa Meio-Norte; 2007.
Carreira CFM. Relação entre a viscosidade in vivo e in vitro de alimentos à base de cevada para frangos de carne [dissertação]. Lisboa: Universidade Técnica de Lisboa; 2011.58 p.

Calsamiglia S, Ferret A, Bach A. Tablas FEDNA de valor nutritivo de forrajes y subproductos fibrosos húmedos. Madri: Fundación para el Desarrollo de la Nutrición Animal; 2016. 93 p.

Costa AD, Mattos ES, Lima CAR, Vieira AA, MatoS MA, Ferreira RA, et al. Composição química e energia digestível do bagaço de malte em suínos machos nas fases de crescimento e terminação. Anais Zootec; 2006; Recife, PE.

Madeira LA, Sartori JR, Araujo PC, Pizzolante CC, Saldanha ESPB, Pezzato AC. Avaliação do desempenho e do rendimento de carcaça de quatro linhagens de frangos de corte em dois sistemas de criação. R Bras Zootec. 2010;39(10):2214-21.

Oliveira EJDN, Lima KRDS, Pereira TS, Pereira RB, Silveira AS, Manno MC. Utilização de resíduo de cervejaria desidratado com duas granulometrias para frango de corte na fase de 1 a 14 dias de idade. Anais Zootec; 24 - 27 maio 2005; Campo Grande, MS.

Parpinelli W. Utilização de resíduo seco de cervejaria na alimentação de frangos de corte [dissertação]. Dois Vizinhos: Universidade Tecnológica Federal do Paraná; 2016. 101 p.

Rosin DP. Composição química e inclusão de cevada, com ou sem adição de xilanase, na ração para frango de corte [dissertação]. Vila Velha: Universidade de Vila Velha; 2012. 50 p.

Rostagno HS, Albino LFT, Donzele JL, Gomes PC, Oliveira RF, Lopes DC, et al. Tabelas brasileiras para aves e suínos: composição de alimentos e exigências nutricionais. Viçosa: Universidade Federal de Viçosa; 2011. 252 p. 